\title{
Association des comorbidités psychiatriques avec la durée de séjour des patients en médecine interne d'aval des Urgences
}

Association between psychiatric comorbidities and hospital length of stay in post-emergency internal medicine patients

A. Lampros (1), C. Montardi (2), L. Journeau (3), S. Georgin-Lavialle (1), T. Hanslik (2), R. Dhôte (3), C. Goujard (4), C. Le Jeunne (5), I. Mahe (3), T. Papo (7), B. Godeau (8), A. Bourgarit (9), O. Fain (10), B. Fantin (11), N. Dzierzynski (12), J. Leblanc (13), C.Nevoret (14), O. Steichen (1)

(1) AP-HP, Sorbonne Université, Médecine interne, Hôpital Tenon, 4 rue de la Chine 75020 Paris;

(2) AP-HP, Médecine interne, Hôpital Ambroise Paré, 9 avenue Charles-de-Gaulle 92104 Boulogne-

Billancourt ;

(3) AP-HP, Médecine interne, Hôpital Louis - Mourier, 178 rue des Renouillers 92701 Colombes;

(4) AP-HP, Médecine interne, Hôpital Avicenne, 125 rue de Stalingrad 93000 Bobigny ;

(5) AP-HP, Médecine interne, Hôpital Bicêtre, 78 rue du Général Leclerc 94275 Le Kremlin-Bicêtre;

(6) AP-HP, Médecine interne, Hôpital Cochin, Paris 27, rue du Faubourg-Saint-Jacques 75014 Paris ;

(7) AP-HP, Médecine interne, Hôpital Bichat - Claude Bernard, 46 rue Henri Huchard 75018 Paris ;

(8) AP-HP, Médecine interne, Hôpital Henri Mondor 51 avenue Mar de Lattre de Tassigny, 94000 Créteil ;

(9) AP-HP, Médecine interne, Hôpital Jean Verdier, Avenue du 14 juillet 93143 Bondy;

(10) AP-HP, Médecine interne, Hôpital Saint Antoine 184 rue Faubourg Saint Antoine, 75012 Paris ;

(11) AP-HP, Médecine interne, Hôpital Beaujon, 100 boulevard du Général Leclerc 92110 Clichy ;

(12) Psychiatrie, Hôpital Tenon 4 rue de la Chine 75020 Paris;

(13) AP-HP, URC-Est, 184 rue Faubourg Saint Antoine, 75012 Paris ; Sorbonne Université, INSERM, Institut Pierre Louis d'Épidémiologie et de Santé Publique ;

(14) AP-HP, Département Web Innovations Données (WIND), direction des systèmes d'information, 33 boulevard de Picpus, 75012 Paris.

Auteur correspondant : Olivier Steichen

Service de médecine interne, hôpital Tenon

4 rue de la Chine, F-75020 Paris

Tel : 0156016077 ; Fax : 0156017146

Email : olivier.steichen@aphp.fr 


\section{Résumé}

\section{Introduction}

Les patients atteints de troubles psychiatriques souffrent de problèmes somatiques nombreux et souvent mal pris en charge. Cette étude vise à établir le profil des patients avec comorbidités psychiatriques hospitalisés en médecine interne depuis les urgences et à comparer leurs durées de séjour avec celles des patients sans comorbidité psychiatrique.

\section{Méthodes}

Cette étude observationnelle rétrospective sur données de soins collectées dans l'Entrepôt de Données de Santé de l'Assistance Publique-Hôpitaux de Paris a inclus les patients hospitalisés après passage aux urgences, au cours de l'année 2017, dans 9 services de médecine interne. L'existence d'une comorbidité psychiatrique et le poids des comorbidités somatiques (score de Charlson) étaient déterminés par le codage diagnostique. Le score de Charlson et la durée moyenne de séjour étaient comparés entre les patients avec et sans comorbidités psychiatriques.

\section{Résultats}

8981 séjours étaient inclus (8 001 patients), dont 1867 (21\%) avec comorbidité psychiatrique. Après ajustement sur l'âge, le sexe, le service et le diagnostic principal (DP), le score de Charlson moyen était supérieur de 0,68 chez les patients avec comorbidités psychiatriques $(\mathrm{p}<0,001)$ et leur durée moyenne de séjour était supérieure de $30 \%$ après ajustement supplémentaire sur le score de Charlson $(\mathrm{p}<0,001)$, correspondant à un. Ces différences étaient similaires dans les analyses restreintes à chaque principale catégorie de DP.

\section{Conclusion}

Les patients avec comorbidités psychiatriques sont nombreux en hospitalisation d'aval des Urgences. Ils ont des durées de séjour plus longues, incomplètement expliquées par des comorbidités somatiques plus lourdes. Une attention particulière doit être portée à cette population vulnérable.

Mots clefs : Troubles mentaux ; comorbidité ; médecine hospitalière ; durée du séjour ; recherche sur les services de santé 


\begin{abstract}

\section{Introduction}

Patients with psychiatric disorders suffer from a higher rate of somatic disorders than those without psychiatric disorder, often inappropriately managed. Our study aimed to describe patients with psychiatric comorbidity in post emergency internal medicine units and to compare their length of hospital stay to patients without psychiatric disease.
\end{abstract}

\title{
Methods
}

This French cross sectional study used the data warehouse of the greater Paris hospitals. It included, all patients hospitalized through the emergency department in 9 internal medicine departments during the year 2017. Psychiatric disorders and the burden of somatic disorders (Charlson score) were determined through diagnostic coding. Charlson score and hospital length of stay were compared between patients with and without psychiatric comorbidity.

\section{Results}

8981 hospital stays (8001 patients) were included, 1867 (21\%) with psychiatric comorbidity. After adjusting for age, gender, hospital and main diagnosis, the Charlson score was on average 0,68 higher in the psychiatric comorbidity group $(\mathrm{p}<0,001)$ and the length of hospital stay was $30 \%$ higher after further adjustment on the Charlson score $(\mathrm{p}<0,001)$. These differences were consistent for each main diagnosis.

\section{Conclusion}

Patients with psychiatric comorbidity are frequent in post emergency internal medicine wards. They experience longer hospital stays, only partly related with a higher burden of somatic disorders. Special attention should be paid to this vulnerable population.

Keywords: Mental disorders; comorbidity; hospital medicine; length of stay; health service research 


\section{Introduction}

\subsection{Contexte}

La prise en charge de la santé mentale représente un enjeu de santé publique par son poids épidémiologique (plus d'un français sur dix concerné en 2016) et économique (14\% des dépenses de l'assurance maladie) [1]. Dix à quinze pourcents de ces dépenses sont en lien avec des hospitalisations en secteur médical ou chirurgical. Les malades psychiatriques ont des problèmes somatiques plus fréquents, plus graves et dont la prise en charge se fait plus fréquemment en hospitalisation non programmée [2].

En effet, les maladies psychiatriques sont associées à de nombreux facteurs d'exposition et de fragilité comme la précarité sociale, l'alcool, le tabac, la sédentarité. Elles sont également associées à un accès moins généralisé aux mesures de prévention et aux soins primaires [3]. Enfin, elles complexifient l'approche diagnostique et thérapeutique des problèmes somatiques [4].

Les troubles psychiatriques sont fréquents chez les patients hospitalisés en médecine interne en raison de leur association aux maladies immuno-inflammatoires (témoignant des liens entre régulation immunitaire et neuropsychiatrique), mais également chez les patients hospitalisés en aval des urgences sur lesquels cette étude se focalise [5].

\subsection{Objectifs de l'étude}

Les objectifs de l'étude étaient d'estimer la prévalence des comorbidités psychiatriques en hospitalisation non programmée en médecine interne après admission par les urgences et d'analyser leur association avec les comorbidités somatiques et la durée de séjour.

\section{Méthodes}

\subsection{Schéma expérimental}

Il s'agit d'une étude observationnelle rétrospective multicentrique réalisée sur données médicoadministratives et données de soins collectées prospectivement dans le système d'information hospitalier et versées dans l'Entrepôt de Données de Santé de l'Assistance Publique-Hôpitaux de Paris (EDS AP-HP). L'article est rédigé conformément à l'énoncé RECORD [6]. 


\subsection{Population étudiée}

L'étude porte sur 9 services de médecine interne de l'AP-HP utilisant le logiciel de dossier médical informatisé Orbis (Agfa) en 2017 : Ambroise Paré, Avicenne, Bichat, Cochin, Henri Mondor, Jean Verdier, Bicêtre, Louis Mourier, Tenon. Tous les séjours concernant des patients majeurs hospitalisés après passage aux urgences du $1^{\mathrm{er}}$ janvier au 31 décembre 2017 ont été inclus. Les séjours erronés (créés sur un doublon d'identité) et les mouvements erronés en médecine interne (créés par erreur) ont été repérés par l'absence de codage diagnostique et exclus.

\subsection{Variables étudiées}

Les données analysées étaient toutes issues de l'EDS AP-HP, aucune autre source de données n'a été utilisée.

L'existence d'une comorbidité psychiatrique a été déterminée par le codage diagnostique du séjour et de toute hospitalisation dans les 365 jours précédents : diagnostic principal (DP), associé ou relié entre F1X et F4X, excepté F17 (addiction au tabac) sans autre code psychiatrique. Les comorbidités psychiatriques ont été catégorisées en 4 groupes selon le codage afin de réaliser des analyses en sousgroupes : troubles psychotiques, troubles de l'humeur (troubles dépressifs et troubles bipolaires), troubles névrotiques (troubles anxieux essentiellement), troubles addictifs (sauf addiction au tabac seule).

Le poids des comorbidités somatiques, estimé par le score de Charlson, a également été déterminé par le codage diagnostique en utilisant la méthode validée pour les données médico-administratives chez les patients hospitalisés [7]. Les DP codés lors du mouvement en médecine interne ont été regroupés selon l'arborescence de la CIM 10, de manière à former des catégories cliniquement pertinentes. La durée de séjour analysée allait de l'arrivée aux Urgences jusqu'à la sortie de l'hôpital, hors soins de suite et réadaptation.

\subsection{Prise en compte des biais}

La présence d'une comorbidité psychiatrique et la valeur du score de Charlson établies par le codage diagnostique ont été évaluées par retour manuel sur un échantillon aléatoire de 140 dossiers issus de trois services, par trois des investigateurs en aveugle du codage. L'existence d'une comorbidité psychiatrique était avérée par la satisfaction d'au moins une des trois conditions suivantes : diagnostic 
par un psychiatre ; suivi par un psychiatre ou un psychologue ; traitement psychotrope au long cours (sauf hypnotiques seuls). L'échantillonnage était stratifié de façon équilibrée par centre, par présence ou absence de comorbidité psychiatrique codée, et par valeur du score de Charlson calculé à partir du codage ( 0,1 ou 2,3 ou 4,5 ou plus).

\subsection{Taille de l'échantillon de patients}

Les données de soins sont majoritairement versées dans l'EDS AP-HP à partir d du dossier patient informatisé Orbis progressivement déployé sur l'ensemble de l'AP-HP depuis 2012 et jusqu'en 2020. L'exhaustivité des données dans l'EDS AP-HP dépend donc du déploiement du logiciel dans l'hôpital et le service concerné. Cette étude a porté sur les données collectées sur une année complète récente, représentant ainsi le recrutement annuel d'un maximum de services de médecine interne utilisant Orbis.

\subsection{Analyses statistiques}

Après avis favorable du Comité Scientifique et Ethique de l'AP-HP, les données ont été mises à disposition sur une grappe de serveurs sécurisés commandés par le système de calcul distribué Spark. Cette procédure permet de traiter les données pseudonymisée sur les serveurs de l'AP-HP au sein d'un

espace de travail sécurisé, dédié à la recherche et avec une puissance de calcul adaptée. Le logiciel R pour Spark (SparkR) a été utilisé pour les analyses.

La concordance entre la catégorisation psychiatrique par le codage diagnostique et par retour manuel au dossier informatisé a été évaluée par le calcul du coefficient kappa de Cohen. La concordance entre le score de Charlson calculé avec le codage diagnostique ou par retour manuel au dossier informatisé a été évaluée par le diagramme de Bland et Altman [8] et le coefficient de corrélation de concordance de Lin [9].

L'unité d'analyse est le séjour hospitalier. Les variables quantitatives sont décrites avec la moyenne et l'écart type ou la médiane et les premier et troisième quartiles. Les variables qualitatives sont décrites en effectifs et pourcentages. L'hétérogénéité de la prévalence des comorbidités psychiatriques entre les dix principales catégories de DP a été évaluée avec un modèle de régression logistique ajusté sur l'âge et le sexe en prenant comme effet aléatoire le centre et la catégorie de DP.

L'association entre comorbidités psychiatriques et score de Charlson a été évaluée avec un modèle linéaire à effet mixte ajusté sur le sexe et l'âge, prenant comme effet aléatoire le patient et le centre sur 
l'ensemble des séjours, puis en prenant comme effet aléatoire le patient, le centre et la catégorie de DP pour les dix principales catégories de DP. L'association entre comorbidités psychiatriques et durée de séjour a été évaluée avec un modèle mixte linéaire généralisé avec distribution négative binomiale négative, ajusté sur le sexe, l'âge et le score de Charlson et prenant comme effet aléatoire le patient et le centre sur l'ensemble des séjours, puis prenant comme effet aléatoire le patient, le centre et la catégorie de DP pour les dix principales catégories de DP.

Les analyses par type de comorbidités psychiatriques ont utilisé les mêmes méthodes et ont en outre été ajustées sur les autres types de comorbidités psychiatriques, car un patient pouvait avoir plusieurs types de comorbidités psychiatriques.

\section{Résultats}

\subsection{Population}

9767 séjours hospitalier étaient éligibles, 786 ont été exclus par absence de codage (pas de codage du tout pour 552 et pas de DP codé lors du mouvement en médecine interne pour 234). Au total 8981 séjours étaient inclus correspondant à 8001 patients, dont 1867 séjours (21\%) dans le groupe avec comorbidités psychiatriques (Figure 1). Selon le codage de ses séjours, un même patient pouvait avoir des séjours avec et des séjours sans comorbidité psychiatrique (et donc être compté dans les deux groupes). Les troubles névrotiques représentaient 647 des séjours avec comorbidité psychiatrique (34\%), les trouble psychotique 251 (13\%), les troubles de l'humeur $858(46 \%)$ et les troubles addictifs $581(31 \%) ; 387$ séjours appartenaient à plusieurs sous-groupes psychiatriques à la fois.

L'âge médian lors du séjour était de 68 ans mais significativement moindre dans le groupe avec comorbidité psychiatrique (Tableau 1, p < 0,001). Les femmes représentaient 51\% des séjours étudiés, sans différence significative entre les deux groupes $(\mathrm{p}=0,10$, Tableau 1$)$. Les catégories de diagnostics principaux (DP) les plus représentées étaient (Tableau 2) : drépanocytose (888 séjours, soit $10 \%$ du total), pneumopathie infectieuse ( $801 ; 9 \%)$, infection urinaire $(460 ; 5 \%)$, insuffisance cardiaque $(321 ; 4 \%)$ et exacerbation de BPCO $(307 ; 3 \%)$. Les 10 catégories majoritaires de DP totalisent $40 \%$ de la population totale. 
3.2. Validation des catégorisations médico-administrative, prévalence des comorbidités psychiatriques La validation a porté sur 140 séjours (60 à Tenon, 40 à Ambroise Paré et 40 à Louis Mourier). L'accord entre la catégorisation psychiatrique par le codage diagnostique et par retour au dossier était de $77 \%$, correspondant à un coefficient kappa de Cohen à 0,54 [IC $95 \%: 0,40 ; 0,68$ ]. Le score de Charlson calculé par le codage diagnostique était en moyenne 0,08 point inférieur au résultat obtenu par retour au dossier [IC $95 \%:-4,6 ;+4,5]$, avec une différence s'accentuant légèrement pour les valeurs élevées (Figure 2). Le coefficient de corrélation de concordance de Lin était à 0,65 [IC $95 \%$ : $0,55 ; 0,75]$.

3.3. Validation des catégorisation médico-administrative, prévalence des comorbidités psychiatriques La prévalence des séjours avec comorbidités psychiatriques variait de 12 \% à 29 \% selon l'hôpital (hétérogénéité significative avec $\mathrm{p}<0,001$ ) et de $14 \%$ (insuffisance cardiaque) à $29 \%$ (drépanocytose) en fonction de la catégorie de DP (Tableau 2, hétérogénéité significative avec $\mathrm{p}<$ $0,001)$.

3.4. Association des comorbidités psychiatriques avec le score de Charlson et la durée de séjour

\subsubsection{Toutes comorbidités psychiatriques confondues}

Dans la population totale, le score de Charlson était en moyenne supérieur de 0,38 chez les patients avec comorbidité psychiatrique, après ajustement sur l'âge, le sexe et le centre ( $p=0,01$, hétérogénéité significative en fonction du centre $\mathrm{p}<0,001)$. Dans la population correspondant aux dix catégories majoritaires de DP, le score de Charlson était en moyenne supérieur de 0,68 chez les patients avec comorbidités psychiatriques, après ajustement sur l'âge, le sexe et le centre et le DP $(p=0,003$, hétérogénéité significative en fonction de la catégorie de DP $\mathrm{p}<0,001$ ).

Dans la population totale, la durée de séjour était en moyenne supérieure de 31\% [IC 95\% : $22 ; 41$ ] chez les patients avec comorbidité psychiatrique, après ajustement sur l'âge, le sexe, le score de Charlson et le centre ( $p<0,001$, hétérogénéité significative en fonction du centre $p<0,001$ ). Dans la population correspondant aux dix principales catégories de DP, la durée moyenne de séjour était également supérieure de 29\% [IC 95\% : $17 ; 41$ ] chez les patients avec comorbidités psychiatriques, après ajustement sur l'âge, le sexe, le score de Charlson, le centre et la catégorie de DP ( $p<0,001$, hétérogénéité significative en fonction de la catégorie de DP p $<0,001$, Tableau 2). Pour certaines 
catégories de DP (insuffisance cardiaque, exacerbation de BPCO, érysipèle, gastro-entérite et colite), les patients avec comorbidité psychiatrique hospitalisés sont beaucoup plus jeunes que ceux sans comorbidité psychiatrique. Ceci explique la durée de séjour plus longue en cas de comorbidité psychiatrique après ajustement sur l'âge, alors que la durée de séjour observée sans ajustement est plus longue chez les patients sans comorbidité psychiatrique.

\subsubsection{Par type de comorbidité psychiatrique}

La catégorisation selon les codes psychiatriques fait apparaître 1480 séjours avec un seul type de comorbidité psychiatrique et 387 séjours avec deux types de comorbidités psychiatriques ou plus. Après ajustement, le score de Charlson était significativement plus élevé avec tous les type de comorbidité psychiatrique hormis les troubles psychotiques $(\mathrm{p}=0,19$, Tableau 3$)$ et la durée de séjour plus longue pour tous les types de comorbidité psychiatriques $(\mathrm{p}<0,001$ pour chaque type, Tableau 3). L'amplitude de la différence de durée de séjour était moindre pour chaque type de comorbidité psychiatrique que pour les comorbidités psychiatriques toutes confondues du fait des patients avec plusieurs types de comorbidité, qui cumulent l'impact de chacune sur la durée de séjour.

\section{Discussion}

\subsection{Résultats clefs}

Les comorbidités psychiatriques sont fréquentes (21\%) chez les patients hospitalisés en médecine interne d'aval des urgences. Elles sont associées de manière significative à des comorbidités somatiques plus lourdes et une durée de séjour restant plus longue de $30 \%$ après ajustement sur le poids des comorbidités somatiques.

\subsection{Limites}

\subsubsection{Population étudiée francilienne}

L'étude concerne la population de la région parisienne avec ses particularités démographiques, notamment une forte prévalence de personnes issues de l'immigration : 75\% des bénéficiaires de l'AME de France [10]; entre 10 et $30 \%$ des sujets nés à l'étranger en fonction des départements [11]. 
Cette situation, facteur de vulnérabilité psychique, est très représentée dans les hôpitaux publics du fait d'un accès souvent restreint au secteur de soins primaire.

Le bassin de population et les orientations propres à chaque service expliquent probablement en partie les différences de prévalence des comorbidités psychiatriques observées entre les services participants. La forte prévalence des patients drépanocytaires est une particularité des services de médecine interne en Ile-de-France (10\% du recrutement en aval des urgences), non superposable au reste de la France. Les patients drépanocytaires ont la plus grande fréquence de comorbidités psychiatriques parmi les 10 catégories de DP majoritaires. Les explications sont multiples et intriquées : épisodes douloureux intenses et récidivants depuis l'enfance, retentissement sur les obligations socioprofessionnelles et familiales, stigmatisation y compris au sein de la communauté d'origine, qualité de vie altérée, traumatismes psychiques liés aux complications aiguës graves, aux passages en réanimation et parfois à l'histoire migratoire difficile, menaces sur l'avenir, exposition récurrente aux opioïdes et risque d'addiction... Les comorbidités psychiatriques sont associées chez ces patients à une augmentation de 20 à 30\% de la durée des hospitalisations en urgence, confirmée par cette étude, mais également à des visites aux Urgences trois fois plus fréquentes et des hospitalisation en urgence deux fois plus fréquentes [12].

\subsubsection{Catégorisation psychiatrique sur données médico-administratives}

L'utilisation de données médico-administratives soulève des questions. La concordance du codage diagnostique avec le retour manuel au dossier est modérée, qu'il s'agisse de la catégorisation psychiatrique ou du score de Charlson. Toutefois, le codage diagnostique présente l'avantage de données exhaustives, facilement accessibles pour tous les séjours et relativement homogènes à l'échelle d'un centre. En revanche, le codage diagnostique ne donne pas un reflet précis de toutes les comorbidités et de leurs nuances à chacune, il est réalisé de façon variable d'un service à l'autre [13]. Ceci peut rendre compte d'une partie des différences de prévalence estimée des comorbidités psychiatriques selon les centres hospitaliers. Les analyses d'association stratifiées par centre n'ont toutefois pas mis en évidence d'hétérogénéité majeure dans l'impact des comorbidités psychiatriques sur la durée de séjour, suggérant que le biais de codage différentiel, s’il existe, est faible. Les problématiques de catégorisation nosologique font partie intégrante de la psychiatrie et expliquent certainement une part de ces difficultés. Toutefois, le travail de validation par retour manuel aux dossiers de la catégorisation des patients avec comorbidité psychiatrique s'est heurté à plusieurs 
écueils : la prescription fréquente de psychotrope sans indication bien définie ; des antécédents psychiatriques souvent mal caractérisés ; un recours trop rare aux psychiatres de liaison pour avérer les problèmes psychiatriques ; l'omission fréquente du psychiatre traitant dans les destinataires des comptes rendus.

\subsubsection{Facteurs de confusion}

De nombreux facteurs de confusion potentiels n'ont pas été mesurés, en particulier des facteurs de vulnérabilités socio-économiques qui contribuent très probablement aux différences observées. La précarité sociale, les problèmes de logement, d'emploi, et d'isolement socio-familial sont plus fréquents dans la population avec comorbidités psychiatriques [14-16]. Or, les durées moyennes de séjour reflètent la gravité ou la complexité de la prise en charge médicale mais également les difficultés de prise en charge sociale [16,17]. La stratification des analyses par centre prenant en charge des bassins de population avec un niveau social plus homogène limite ce biais. Néanmoins, ces facteurs contribuent certainement en partie aux différences observées entre patients avec et sans comorbidité psychiatrique.

\subsection{Interprétation}

\subsubsection{Prévalence des comorbidités psychiatriques}

La prévalence des troubles psychiatriques chez les patients hospitalisés a été évaluée dans quelques études trouvant des valeurs proches des nôtres, de l'ordre de 20 à $40 \%$ selon l'approche méthodologique. Une étude danoise réalisée sur 547 patients hospitalisés en médecine interne a évalué l'existence d'un trouble psychiatrique par entretien systématique avec des psychiatres. En se basant sur des critères diagnostiques du DSM elle rapporte une prévalence de l'ordre de $35 \%$ [19]. Cette étude détecte plus de troubles psychiatriques probablement du fait des entretiens systématiques, ce qui illustre la part importante de sous-diagnostic de ces maladies. Une étude réalisée au CHU de Nice analysant le codage diagnostique de tous les patients hospitalisés en court séjour sur l'intégralité de l'hôpital (médecine, chirurgie et gynécologie-obstétrique) durant l'année 2013 trouve une prévalence de $20 \%$ [20]. Cette étude est méthodologiquement proche de la nôtre et trouve une prévalence très similaire de comorbidités psychiatriques. 


\subsubsection{Association avec les comorbidités somatiques}

Nous avons observé une prévalence plus forte de comorbidités somatiques chez les patients atteints de troubles psychiatriques. Il s'agit d'une notion bien décrite, particulièrement frappante pour les maladies cardio-vasculaire et néoplasiques, avec par exemple un risque d'événement cardiovasculaire aigu ou de cancer pulmonaire multiplié par 2 à 3 [1] .

Les facteurs expliquant ce préjudice sont multiples et difficiles à analyser. Plusieurs études suggèrent que les maladies psychiatriques (notamment les troubles de l'humeur) peuvent avoir des conséquences somatiques directes, par dysrégulation neuro-hormonale, entretenant notamment un état inflammatoire chronique à bas bruit, déjà connu comme facteur de risque cardiovasculaire [21]. Toutefois, des facteurs indirects ont probablement un impact prépondérant, comme les effets métaboliques des traitements psychotropes [22] et la surreprésentation des troubles addictifs, notamment de l'exposition alcoolo-tabagique [23].

L'excès de comorbidités somatiques est également favorisé par des différences de prise en charge associées à la présence de comorbidités psychiatriques. Ces différences de prise en charge sont bien étayées en soins primaires. Les patients psychiatriques bénéficient plus rarement des mesures de prévention [3], notamment du dépistage des cancers mammaires et colorectaux avec pour conséquence une prise en charge plus tardive, à un stade avancé de la maladie [23]. En France, 15\% des patients hospitalisés pour trouble psychotique, bipolaire ou dépressif majeur n'ont pas de médecin traitant déclaré au moment de leur hospitalisation [11]. Les malades psychiatriques sont souvent insuffisamment traités pour leurs problèmes de santé somatiques [25]. Ils sont certainement plus difficiles à suivre mais l'existence d'une comorbidité psychiatrique modifie également la manière dont raisonnent les médecins. Une étude américaine sur des vignettes cliniques adressées à 200 médecins généralistes montre que l'appréciation de la gravité, les hypothèses diagnostiques et la réalisation d'examens complémentaires étaient significativement différentes si l'on introduisait la notion d'un antécédent dépressif chez le malade, toutes choses égales par ailleurs [26].

\subsubsection{Impact sur la durée de séjour}

L'association entre la présence d'une maladie psychiatrique et l'allongement de 20 à $30 \%$ de la durée d'hospitalisation trouvée dans notre étude est cohérente avec les données publiées [27]. Une étude réalisée dans un service d'oncologie du Massachusetts ayant inclus prospectivement 1036 patients hospitalisés de façon non programmée dans un contexte de cancer à un stade avancé montre un 
allongement de $18 \%$ de la durée de séjour chez les patients souffrant de dépression. Cette différence n'est pas significative dans le sous-groupe de patients traités par antidépresseurs, ce qui suggère un bénéfice de la prise en charge spécifique [28]. Une étude anglaise réalisée sur les données de codage de 31760 patients hospitalisés pour insuffisance cardiaque trouve un allongement significatif de la durée d'hospitalisation (+30\%) quel que soit le diagnostic psychiatrique codé en comorbidité [24]. Dans notre étude la différence de durée d'hospitalisation reste statistiquement et cliniquement significative après ajustement sur le score de Charlson. Ceci suggère qu'elle n'est pas expliquée simplement par des comorbidités somatiques plus lourdes. Comme évoqué précédemment, des facteurs socio-économiques non mesurés dans l'étude participent certainement, compliquant le retour à domicile, le transfert en soins de suite et de réadaptation.

Il existe peu de données concernant l'impact des comorbidités psychiatriques sur la qualité des soins en milieu hospitalier, mais il est tout à fait possible que cette différence de durée de séjour reflète une prise en charge de moins bonne qualité [2]. Cette hypothèse est souvent avancée, notamment dans les services d'accueil des urgences mais il est logique que les disparités de prise en charge documentées partout ailleurs soient également présentes dans les autres services. Ainsi, les différences de qualité ont été objectivées dans la prise en charge hospitalière de l'infarctus du myocarde et expliquent en partie l'excès de mortalité dont souffrent les patients avec une comorbidité psychiatrique [28].

\subsection{Perspectives}

\subsubsection{Amélioration de la prise en charge des patients avec comorbidité psychiatrique}

La prise en compte des comorbidités psychiatriques se confronte actuellement à des difficultés croissantes générées par la sur-spécialisation et la filiarisation des disciplines médicales. La psychiatrie de liaison s'est développée dans ce contexte. Les anglo-saxons parlent de psychiatrie de « consultation et liaison » reflétant le double rôle de ces équipes : donner un avis diagnostique et thérapeutique en secteur médico-chirurgical mais également assurer la transition avec les structures psychiatriques de ville et organiser le suivi qui fait fréquemment défaut. En effet, $40 \%$ des patients hospitalisés pour troubles psychotiques, bipolaires ou dépressifs n'ont pas de suivi psychiatrique dans l'année qui suit leur hospitalisation [11].

Les équipes psychiatriques de liaison interviennent dans seulement 2 à $4 \%$ des hospitalisations de patients présentant des comorbidités psychiatriques [30-33]. Or, plusieurs études ont trouvé un 
bénéfice à une intervention précoce de ces équipes psychiatriques en médecine et en chirurgie, montrant notamment une réduction de la durée d'hospitalisation [34]. Pour toutes ces raisons, on peut penser que l'intervention plus systématique et précoce des équipes psychiatriques de liaison apporterait de nombreux bénéfices.

Malheureusement, d'autres études montrent une sous-estimation des problèmes psychiatriques et de leurs conséquences par les médecins somaticiens [30,33,35]. Le sous-diagnostic contrevient évidemment à l'appel des psychiatres de liaison. Le problème est particulièrement marqué pour les troubles anxieux et dépressifs, dont plus de $50 \%$ ne sont pas identifiés en hospitalisation. Il semble donc souhaitable d'encourager une formation plus approfondie des internistes à la prise en compte efficace et non stigmatisante des comorbidités psychiatriques.

\subsubsection{Etudes plus approfondies des trajectoires de soins}

Les bases de données hospitalières cliniques et médico-administratives, comme l'EDS AP-HP, et les données nationales de remboursement de soins, comme le Système National des Données de Santé (SNDS) permettraient d'étudier plus finement les trajectoires de soins des patients avec et sans comorbidités psychiatriques. Il serait possible de tester différentes hypothèses pour expliquer leur durée de séjour globale plus longue. On pourrait par exemple imaginer un passage plus long aux Urgences par difficulté d'obtenir un aval en service de spécialité, suivi d'une orientation par défaut en médecine interne. On pourrait également faire l'hypothèse que les patients avec comorbidités psychiatriques nécessitent plus souvent l'orientation en SSR ou la mise en place d'aides à domicile, dont l'organisation allongerait la fin du séjour. La fréquence des réhospitalisations et son association avec la densité du suivi psychiatrique en ville pourraient également être évalués.

\section{Conclusion}

Les maladies psychiatriques sont fréquentes en population hospitalière et sont associées à des comorbidités somatiques nombreuses et une prise en charge plus longue même après ajustement sur le surcroît de comorbidités somatiques. Ceci nous invite à porter une attention particulière à cette population vulnérable dont la santé est touchée dans toutes ses facettes. Une approche globale nécessite de renforcer les interactions avec les équipes psychiatriques de liaison pour articuler toutes les dimensions de prise en charge : somatique et psychiatrique, hospitalière et extra hospitalière, médicale et sociale. 


\section{Financement}

Aucun

\section{Remerciement}

Equipe Web Innovation Données (WIND) de la Direction des Systèmes d'Information pour l'administration de l'Entrepôt de Données de Santé de l'Assistance Publique-Hôpitaux de Paris et pour les réponses rapides et précises aux questions techniques posées par son exploitation.

\section{Conflit d'intérêts}

Aucun 


\section{Bibliographie}

[1] CNAM. Améliorer la qualité du système de santé et maîtriser les dépenses Propositions de 1'Assurance Maladie pour 2019.pdf. ameli.fr 2018.

https://www.ameli.fr/fileadmin/user_upload/documents/rapport-charges-et-produits2019_cp19_01.pdf

[2] Mitchell AJ, Malone D, Doebbeling CC. Quality of medical care for people with and without comorbid mental illness and substance misuse: systematic review of comparative studies. $\mathrm{Br} \mathbf{J}$ Psychiatry 2009;194:491-9

[3] Druss BG, Rosenheck RA, Desai MM, Perlin JB. Quality of preventive medical care for patients with mental disorders. Med Care 2002;40:129-36.

[4] Prince M, Patel V, Saxena S, Maj M, Maselko J, Phillips MR, et al. No health without mental health. Lancet 2007;370:859-77.

[5] Saliou V, Fichelle A, McLoughlin M, Thauvin I, Lejoyeux M. Psychiatric disorders among patients admitted to a French medical emergency service. Gen Hosp Psychiatry 2005;27:263-8.

[6] Benchimol EI, Smeeth L, Guttmann A, Harron K, Moher D, Petersen I, et al. The REporting of studies Conducted using Observational Routinely-collected health Data (RECORD) Statement. PLoS Med 2015;12.

[7] Quan H, Li B, Couris CM, Fushimi K, Graham P, Hider P, et al. Updating and validating the Charlson comorbidity index and score for risk adjustment in hospital discharge abstracts using data from 6 countries. Am J Epidemiol 2011;173:676-82

[8] Bland JM, Altman DG. Statistical methods for assessing agreement between two methods of clinical measurement. Lancet 1986;1:307-10.

[9] Lawrence I-Kuei Lin. A Note on the Concordance Correlation Coefficient. Biometrics 2000;56:324-5.

[10] Drhil, Service des Observatoires,, des Études et de l’Évaluation. Pauvreté, précarité, hébergement, logment : les données de référence en ile de france au 31.12.2010 https://drees.solidarites-sante.gouv.fr/etudes-et-statistiques/technique/publicationsregionales/article/ile-de-france.

[11] Direction de la recherche, des études, de l'évaluation et des statistiques (DREES). Enquête de la DREES et de l’ IRDES “Atlas de la santé mentale en France” 2018 
http://santementale.atlasante.fr/\#s=2013;z=579472,7010857,2335916,726451;l=fr;i=cont_agreg_ 04.ter_06;v=map3.

[12] Fayand A, Dzierzynski N, Georgin-Lavialle S, Bachmeyer C, Mattioni S, Stankovic-Stojanovic K, Lionnet F, Steichen O. The association of psychiatric comorbidities with emergency visits and hospitalisations in adult sickle-cell patients: a cohort study. Br J Haematol 2019 [in press]

[13] Haut conseil de santé publique. Pour une meilleure utilisation des bases de données nationales pour la santé publique et la recherche 2012.

[14] Haut conseil de santé publique. La progression de la précarité en France et ses effets sur la santé 2007.

[15] Fazel S, Khosla V, Doll H, Geddes J. The Prevalence of Mental Disorders among the Homeless in Western Countries: Systematic Review and Meta-Regression Analysis. PLoS Med 2008;5:e225.

[16] Laporte A, Le Mener E, Detrez MA, Douay C, Le Strat Y, Vandentorren S. La santé mentale et les addictions chez les personnes sans logement personnel en Île-de-France: l'enquête Samenta de 2009. Numéro thématique. État de santé et conditions de vie des populations sans domicile. Bull Epidemiol Hebd 2015;36-37.

[17] Yilmaz E, Raynaud D. Les patients en situation de précarité accueillis en court séjour restent-ils plus longtemps à l'hôpital ? Le panorama des établissements de santé (2010). http://fulltext.bdsp.ehesp.fr/Ministere/Drees/Publications/2011/etabsante2010-patient.pdf.

[18] Mathy C, Bensadon M. Le surcoût de l'hospitalisation des patients précaires. J Econ Méd 2002;20:263-78.

[19] Hansen MS, Fink P, Frydenberg M, Oxhøj M-L, Søndergaard L, Munk-Jørgensen P. Mental disorders among internal medical inpatients: Prevalence, detection, and treatment status. J Psychosom Res 2001;50:199-204.

[20] Bourdais-Mannone C, Gendreike Y, Clamen A, Teadi J, Jover F, David R, et al. Fréquence et codage des prises en charge psychiques des patients hospitalisés en court séjour. Santé Publique 2015;27:221-31.

[21] Halaris A. Inflammation-Associated Co-morbidity Between Depression and Cardiovascular Disease. Curr Top Behav Neurosci 2017;31:45-70. 
[22] Nielsen R-E, Lolk A, M Rodrigo-Domingo null, Valentin J-B, Andersen K. Antipsychotic treatment effects on cardiovascular, cancer, infection, and intentional self-harm as cause of death in patients with Alzheimer's dementia. Eur Psychiatry J 2017;42:14-23.

[23] Hjorthøj C, Østergaard MLD, Benros ME, Toftdahl NG, Erlangsen A, Andersen JT, et al. Association between alcohol and substance use disorders and all-cause and cause-specific mortality in schizophrenia, bipolar disorder, and unipolar depression: a nationwide, prospective, register-based study. Lancet Psychiatry 2015;2:801-8.

[24] Wadia RJ, Yao X, Deng Y, Li J, Maron S, Connery D, et al. The effect of pre-existing mental health comorbidities on the stage at diagnosis and timeliness of care of solid tumor malignances in a Veterans Affairs (VA) medical center. Cancer Med 2015;4:1365-73.

[25] Mitchell AJ, Lord O, Malone D. Differences in the prescribing of medication for physical disorders in individuals with v. without mental illness: meta-analysis. Br J Psychiatry 2012;201:435-43.

[26] Graber MA, Bergus G, Dawson JD, Wood GB, Levy BT, Levin I. Effect of a Patient's Psychiatric History on Physicians' Estimation of Probability of Disease. J Gen Intern Med 2000;15:204-6.

[27] Jansen L, van Schijndel M, van Waarde J, van Busschbach J. Health-economic outcomes in hospital patients with medical-psychiatric comorbidity: A systematic review and meta-analysis. Plos One 2018;13:e0194029.

[28] Wong RL, El- Jawahri A, D’Arpino SM, Fuh C-X, Johnson PC, Lage DE, et al. Use of Antidepressant Medications Moderates the Relationship Between Depressive Symptoms and Hospital Length of Stay in Patients with Advanced Cancer. The Oncologist 2019;24:117-24.

[29] Druss BG, Bradford WD, Rosenheck RA, Radford MJ, Krumholz HM. Quality of Medical Care and Excess Mortality in Older Patients With Mental Disorders. Arch Gen Psychiatry 2001;58:565-72.

[30] Gous P, Gagiano CA, Weich DJ. Psychiatric disorders in internal medicine. South Afr Med J Suid-Afr Tydskr Vir Geneeskd 1992;82:441-2.

[31] Silverstone PH. Prevalence of psychiatric disorders in medical inpatients. J Nerv Ment Dis 1996; 184:43-51.

[32] De AK, Kar P. Psychiatric disorders in medical in patients A study in a teaching hospital. Indian J Psychiatry 1998;40:73-8. 
[33] Seltzer A. Prevalence, detection and referral of psychiatric morbidity in general medical patients. J R Soc Med 1989;82:410-2.

[34] Oldham MA, Chahal K, Lee HB. A systematic review of proactive psychiatric consultation on hospital length of stay. Gen Hosp Psychiatry 2019;60:120-6.

[35] Al-Huthail YR. Accuracy of Referring Psychiatric Diagnosis. Int J Health Sci 2008;2:35-8.

Figure 1. Population incluse dans l'étude

Figure 2. Diagramme de Bland et Altman du score de Charlson calculé à partir du codage diagnostique ou par retour au dossier

Tableau 1. Caractéristiques de la population étudiée et des séjours avec ou sans comorbidité psychiatrique

Tableau 2. Nombre de séjours dans les dix principales catégories de diagnostics principaux (DP), proportion avec comorbidité psychiatrique et durées moyennes de séjour avec ou sans comorbidité psychiatrique

Tableau 3. Différence du score de Charlson et de la durée de séjour entre les patients avec et sans chaque type de comorbidité psychiatrique 
Tableau 1. Caractéristiques de la population étudiée et des séjours avec ou sans comorbidité psychiatrique

\begin{tabular}{|l|c|c|c|}
\hline & $\begin{array}{c}\text { Séjours avec } \\
\text { comorbidité } \\
\text { psychiatrique } \\
(\mathbf{n = 1 8 6 7 )}\end{array}$ & $\begin{array}{c}\text { Séjours sans } \\
\text { comorbidité } \\
\text { psychiatrique } \\
(\mathbf{n = 7 1 1 5 )}\end{array}$ & $\mathbf{p}$ \\
\hline Femmes & $976(52 \%)$ & $3566(50 \%)$ & 0,10 \\
\hline Âge (années) & $63[44 ; 91]$ & $69[47 ; 92]$ & $<0,001$ \\
\hline Score de Charlson & $1[0 ; 3]$ & $1[0 ; 3]$ & $0,01^{*}$ \\
\hline Durée de séjour & $9[5 ; 16]$ & $7[4 ; 13]$ & $<0,001^{* *}$ \\
(jours) & & & \\
\hline
\end{tabular}

Résultats exprimés sous forme de nombre (pourcentage) ou médiane [quartiles]

* ajusté sur âge, sexe et centre

** ajusté sur âge, sexe, centre et score de Charlson 
Tableau 2. Nombre de séjours dans les dix principales catégories de diagnostics principaux (DP), proportion avec comorbidité psychiatrique et durées médianes de séjour [quartiles] avec ou sans comorbidité psychiatrique

\begin{tabular}{|c|c|c|c|c|c|}
\hline \multirow[t]{2}{*}{ Catégorie de DP } & \multirow[t]{2}{*}{$\begin{array}{l}\text { Nombre de séjours } \\
\text { (\% du total des } \\
\text { séjours) }\end{array}$} & \multirow{2}{*}{$\begin{array}{l}\text { Nombre de séjours } \\
\text { avec comorbidité } \\
\text { psychiatrique } \\
\text { (\% de la catégorie) }\end{array}$} & \multicolumn{2}{|c|}{$\begin{array}{c}\text { Durée de séjour selon la présence } \\
\text { d'une comorbidité psychiatrique } \\
\text { (jours) }\end{array}$} & \multirow[t]{2}{*}{$\begin{array}{l}\text { Différence relative } \\
\text { de durée de séjour } \\
\text { [IC 95\% ]* }\end{array}$} \\
\hline & & & avec & sans & \\
\hline Drépanocytose & $888(9,9 \%)$ & $260(29 \%)$ & $6[3 ; 9]$ & $5[3 ; 8]$ & $+27 \%[5 ; 54]$ \\
\hline Pneumopathie & $801(8,9 \%)$ & $170(21 \%)$ & $11[6,25 ; 19]$ & $8[5 ; 13]$ & $+36 \%[13 ; 63]$ \\
\hline Infection urinaire & $460(5,1 \%)$ & $73(16 \%)$ & $9[5 ; 15]$ & $6[4 ; 10]$ & $+26 \%[4 ; 53]$ \\
\hline Insuffisance cardiaque & $321(3,6 \%)$ & $44(14 \%)$ & $9[6 ; 15,5]$ & $10[6 ; 16]$ & $+23 \%[1 ; 50]$ \\
\hline \begin{tabular}{|l|} 
Exacerbation de \\
BPCO
\end{tabular} & $307(3,4 \%)$ & $77(25 \%)$ & $7[4,25 ; 13]$ & $8[5,5 ; 13]$ & $+27 \%[5 ; 55]$ \\
\hline Thrombose veineuse & $274(3,1 \%)$ & $40(15 \%)$ & $11[5 ; 18,25]$ & $7[4 ; 12]$ & $+33 \%[9 ; 62]$ \\
\hline Erysipèle & $204(2,3 \%)$ & $32(16 \%)$ & $7[4,25 ; 13]$ & $8[5,5 ; 13]$ & $+24 \%[1-52]$ \\
\hline Anémie ferriprive & $154(1,7 \%)$ & $27(17 \%)$ & $10[5,5 ; 18,5]$ & $7[4 ; 12]$ & $+29 \%[5 ; 58]$ \\
\hline AVC & $138(1,5 \%)$ & $20(14 \%)$ & $11[6,75 ; 21,25]$ & $9[5 ; 17,5]$ & $+32 \%[7 ; 62]$ \\
\hline $\begin{array}{l}\text { Gastro entérite et } \\
\text { colite }\end{array}$ & $136(1,5 \%)$ & $27(20 \%)$ & $5[4 ; 16,5]$ & $6[3 ; 9]$ & $+31 \%[6 ; 60]$ \\
\hline
\end{tabular}

Résultats exprimés sous forme de nombre (pourcentage) ou médiane [quartiles]

* ajustée à l'âge, au sexe et au score de Charlson 
Tableau 3. Différence du score de Charlson et de la durée de séjour entre les patients avec et sans chaque type de comorbidité psychiatrique

\begin{tabular}{|l|c|c|}
\hline & $\begin{array}{c}\text { Différence de score de } \\
\text { Charlson }[\text { IC 95\% }\end{array}$ & $\begin{array}{c}\text { Différence relative de durée } \\
\text { de séjour }[\text { IC 95\% }\end{array}$ \\
\hline Troubles névrotiques $(\mathbf{n}=\mathbf{6 4 7})$ & $+0,38[0,18 ; 0,57]$ & $+20 \%[12 ; 28]$ \\
\hline Troubles psychotiques $(\mathbf{n}=\mathbf{2 5 1})$ & $+0,19[-0,09 ;+0,48]$ & $+21 \%[10 ; 34]$ \\
\hline Troubles de l'humeur $(\mathbf{n}=\mathbf{8 5 8})$ & $+0,37[0,20 ; 0,53]$ & $+15 \%[8 ; 22]$ \\
\hline Troubles addictifs $(\mathbf{n}=\mathbf{5 8 1})$ & $+0,30[0,10 ; 0,50]$ & $+33 \%[23 ; 42]$ \\
\hline
\end{tabular}

* ajusté sur âge, sexe, centre et autres types de comorbidité psychiatrique

** ajusté sur âge, sexe, centre, autres types de comorbidité psychiatrique et score de Charlson 